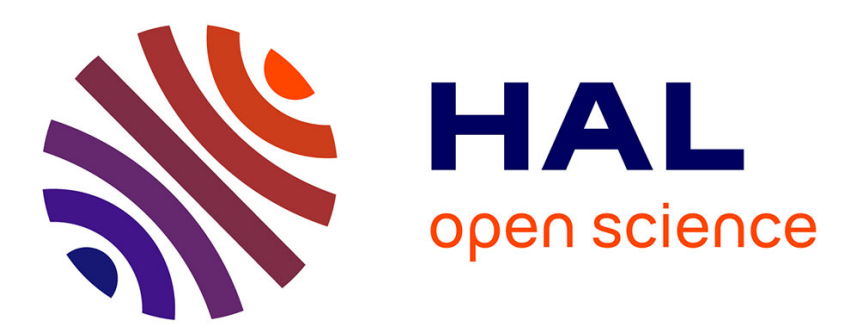

\title{
Revisiting the iISS small-gain theorem through transient plus ISS small-gain regulation
}

Hiroshi Ito, Randy Freeman, Antoine Chaillet

\section{To cite this version:}

Hiroshi Ito, Randy Freeman, Antoine Chaillet. Revisiting the iISS small-gain theorem through transient plus ISS small-gain regulation. Asian Journal of Control, 2013, 15 (1), pp.11-19. 10.1002/asjc.538 . hal-00780483

\section{HAL Id: hal-00780483 \\ https://hal-centralesupelec.archives-ouvertes.fr/hal-00780483}

Submitted on 24 Jan 2013

HAL is a multi-disciplinary open access archive for the deposit and dissemination of scientific research documents, whether they are published or not. The documents may come from teaching and research institutions in France or abroad, or from public or private research centers.
L'archive ouverte pluridisciplinaire HAL, est destinée au dépôt et à la diffusion de documents scientifiques de niveau recherche, publiés ou non, émanant des établissements d'enseignement et de recherche français ou étrangers, des laboratoires publics ou privés. 


\title{
REVISITING THE IISS SMALL-GAIN THEOREM THROUGH TRANSIENT PLUS ISS SMALL-GAIN REGULATION
}

\author{
Hiroshi Ito, Randy A. Freeman, and Antoine Chaillet
}

\begin{abstract}
Recently, the small-gain theorem for input-to-state stable (ISS) systems has been extended to the class of integral input-to-state stable (iISS) systems. Feedback connections of two iISS systems are robustly stable with respect to disturbance if an extended small-gain condition is satisfied. It has been proved that at least one of the two iISS subsystems needs to be ISS for guaranteeing globally asymptotic stability and iISS of the overall system. Making use of this necessary condition for the stability, this paper gives a new interpretation to the iISS small gain theorem as transient plus ISS small-gain regulation. The observation provides useful information for designing and analyzing nonlinear control systems based on the iISS small-gain theorem.
\end{abstract}

Key Words: Integral input-to-state stability, nonlinear interconnected systems, small gain theorem.

\section{INTRODUCTION}

For analysis and design of nonlinear systems the ISS small-gain theorem has been widely used [12,17]. The theorem covers the class of input-to-state stable (ISS) systems and answers the question of whether their feedback interconnection is again ISS. It was first proved with a trajectorybased approach in [12]. A version relying on the Lyapunov functions associated with each of the subsystems was subsequently presented in [11]. While the construction of a Lyapunov function for the overall interconnection is useful from the analysis and design viewpoints, the trajectory-based proof is simpler and illustrates more intuitively the idea of contraction. Recently, the small-gain theorem has been extended to the interconnection of integral input-to-state stable (iISS) systems in $[6,10]$. The iISS is a more general robustness property than ISS $[14,15]$, and the theorem in $[6,10]$ includes the ISS small-gain theorem as a special case. In these references, a Lyapunov function is explicitly constructed for the overall interconnection. Another approach, developed in [1], makes use of monotonicity and nullclines in verifying that the equilibrium of the interconnection of iISS systems is globally asymptotically stable (GAS). Although the approach proposed there does not apply to systems with exogenous inputs,

Manuscript received July 15, 2011; revised January 4, 2012; accepted March 14, 2012.

H. Ito is with Department of Systems Design and Informatics, Kyushu Institute of Technology, 680-4 Kawazu, Iizuka 820-8502, Japan (e-mail: hiroshi@ces.kyutech.ac.jp).

R.A. Freeman is with Department of Electrical Engineering and Computer Science, Northwestern University, Evanston, IL 60208-3118 USA (e-mail: freeman@ eecs.northwestern.edu).

A. Chaillet is with L2S, EECI, Univ. Paris Sud 11 - Supélec, 3 rue Joliot Curie, 91192 Gif-sur-Yvette, France (e-mail: chaillet@ieee.org).

The work is supported in part by a Grant-in-Aid for Scientific Research (C) of JSPS under grant numbers 19560446 and 22560449. it offers a useful interpretation of GAS for feedback connections of iISS and ISS subsystems.

This paper revisits the iISS small-gain theorem and gives an insight into its mechanism. It also gives an interpretation which connects the iISS small-gain theorem with the contractive behavior of trajectories explained by the standard ISS small-gain theorem. In order to understand how the trajectories of interconnected systems evolve, this paper assumes that iISS dissipation inequalities are given for both individual subsystems. As illustrated by the result in [5] on cascaded iISS systems, the use of dissipation inequalities of subsystems is more successful than using trajectory bounds when dealing with interconnected iISS systems. This paper follows this idea to tackle feedback interconnected systems. The proof this paper develops splits the system trajectory into a transient response and a subsequent response governed by the ISS small-gain condition. This paper illustrates how this strategy enables us to deal with iISS systems which are not ISS.

Notations. The Euclidean norm of a real vector on $\mathbb{R}^{n}$ is denoted by the symbol $\mid \cdot$. A continuous function $\omega: \mathbb{R}_{+}:=$ $[0, \infty) \rightarrow \mathbb{R}_{+}$is said to be positive definite and denoted by $\omega \in$ $\mathcal{P}$ if it satisfies $\omega(0)=0$ and $\omega(s)>0$ holds for all $s>0$. A function is of class $\mathcal{K}$ if it belongs to $\mathcal{P}$ and is strictly increasing; of class $\mathcal{K}_{\infty}$ if it is of class $\mathcal{K}$ and is unbounded. A continuous function $\beta: \mathbb{R}_{+} \times \mathbb{R}_{+} \rightarrow \mathbb{R}_{+}$is of class $\mathcal{K} \mathcal{L}$ if $\beta(\cdot, t)$ is of class $\mathcal{K}$ for each $t \geq 0$, and $\beta\left(s,{ }^{\circ}\right)$ is non-increasing and goes to zero as $t \rightarrow \infty$ for each $s \geq 0$. The identity map on $\mathbb{R}$ is denoted by Id. If $\omega$ is a class $\mathcal{K}_{\infty}$ function, its inverse $\omega^{-1}$ is of class $\mathcal{K}_{\infty}$. For $\omega \in \mathcal{K} \backslash \mathcal{K}_{\infty}$, its inverse $\omega^{-1}$ is defined on the finite interval $\left[0, \lim _{\tau \rightarrow \infty} \omega(\tau)\right)$ since the continuous function $\omega$ is strictly increasing and $\omega(0)=0$. Following the convention employed in $[6,10]$, in this paper, $s<\lim _{\tau \rightarrow \infty} \omega(\tau)$ or 
$\infty=\lim _{\tau \rightarrow \infty} \omega(\tau)$ is implied whenever $\omega^{-1}(s)$ is used. For a function $\gamma \in \mathcal{P}$, we write $\gamma \in \mathcal{O}(>L)$ with a non-negative number $L$ if there exists a positive number $K>L$ such that lim $\sup _{\mathrm{s} \rightarrow 0} \gamma(s) / s^{K}<\infty$ holds. We write $\gamma \in \mathcal{O}(L)$ when $K=L$. The symbols $\vee$ and $\wedge$ denote logical sum and logical product, respectively. For $f, g: \mathbb{R}_{+} \rightarrow \mathbb{R}_{+}$, we use the simple notation $\lim f(s)=\lim g(s)$ to describe $\{\lim f(s)=\infty \wedge \lim$ $g(s)=\infty\} \vee\{\infty>\lim f(s)=\lim g(s)\}$. Note that the $\infty$ case is included. In a similar manner, $\lim f(s) \geq \lim g(s)$ denotes $\{\lim f(s)=\infty \vee \infty>\lim f(s) \geq \lim g(s)\}$. A system $\dot{x}=f(x)$ admitting a unique maximal solution $x(t) \in \mathbb{R}^{n}$ for any initial condition $x(0) \in \mathbb{R}^{n}$ is said to be GAS if its origin is globally asymptotically stable. We let $\mathcal{U}$ denote the set of all measurable locally essentially bounded signals $u: \mathbb{R}_{+} \rightarrow \mathbb{R}^{m}$. A system $\dot{x}=f(x, u)$ admitting a unique solution $x(t)$ on $\mathbb{R}^{n}$ for any initial condition $x(0) \in \mathbb{R}^{n}$ and any $u \in \mathcal{U}$ is said to have the Bounded Energy Frequently Bounded State (BEFBS, [2]) property with respect to input $u$ and state $x$ if there exists $\sigma \in \mathcal{K}_{\infty}$ such that, if $\int_{0}^{\infty} \sigma(|u(\tau)|) d \tau<\infty$ then $\lim _{\inf _{t \rightarrow \infty}|x(t)|<}$ $\infty$ for all initial conditions $x(0)$. We will make a slight abuse of sup, limsup and inf, liminf to mean the essential supremum and infimum, respectively, where appropriate. A system $\dot{x}=f(x, u)$ is said to be iISS with respect to $u$ if there exist $\chi \in \mathcal{K}_{\infty}, \beta \in \mathcal{K} \mathcal{L}$ and $\gamma \in \mathcal{K}$ such that, for any $x(0) \in \mathbb{R}^{n}$ and any $u \in \mathcal{U}$, a unique solution $x(t) \in \mathbb{R}^{n}$ exists for all $t \geq 0$ and furthermore it satisfies $\chi(|x(t)|) \leq \beta(|x(0)|, t)+\int_{0}^{t} \gamma(|u(\tau)|) d \tau$. A system $\dot{x}=f(x, u)$ is said to be ISS with respect to $u$ if there exist $\beta \in \mathcal{K} \mathcal{L}$ and $\gamma \in \mathcal{K}$ such that, for any $x(0) \in \mathbb{R}^{n}$ and any $u \in \mathcal{U}$, a unique solution $x(t) \in \mathbb{R}^{n}$ exists for all $t \geq 0$ and furthermore it satisfies $\mid x(t) \leq \beta(|x(0)|, t)+\gamma\left(\sup _{\tau \in[0, t]}|\mu(\tau)|\right)$. These are standard definitions borrowed from $[14,15,3]$.

A preliminary version of the material in this paper was presented at the 49th IEEE Conference on Decision and Control [9]. Some errors have been corrected, and the results are refined further in this paper.

\section{A REVIEW OF IISS SMALL-GAIN THEOREM}

Consider the following interconnected system:

$$
\Sigma:\left\{\begin{array}{l}
\Sigma_{1}: \dot{x}_{1}=f_{1}\left(x_{1}, x_{2}, r_{1}\right) \\
\Sigma_{2}: \dot{x}_{2}=f_{2}\left(x_{1}, x_{2}, r_{2}\right)
\end{array}\right.
$$

where $\quad x_{i}(t) \in \mathbb{R}^{n_{i}}, \quad r_{i}(t) \in \mathbb{R}^{m_{i}}, \quad x=\left[x_{1}^{T}, x_{2}^{T}\right]^{T} \in \mathbb{R}^{n} \quad$ and $r=\left[r_{1}^{T}, r_{2}^{T}\right]^{T} \in \mathbb{R}^{m}$. In addition to the existence of a unique maximal solution $x(t)$ for any initial condition $x(0) \in \mathbb{R}^{n}$ and any measurable, locally essentially bounded external input $r$, we assume that the two subsystems satisfy the following dissipation inequalities:
Assumption 1. For each $i \in\{1,2\}$, there exist a continuously differentiable, positive definite and radially unbounded function $V_{i}: x_{i} \in \mathbb{R}^{n_{i}} \rightarrow \mathbb{R}_{+}$and class $\mathcal{K}$ functions $\alpha_{i}, \sigma_{i}, \sigma_{r i}$ such that

$$
\begin{aligned}
& \dot{V}_{1} \leq-\alpha_{1}\left(V_{1}\left(x_{1}\right)\right)+\sigma_{1}\left(V_{2}\left(x_{2}\right)\right)+\sigma_{r 1}\left(\left|r_{1}\right|\right) \\
& \dot{V}_{2} \leq-\alpha_{2}\left(V_{2}\left(x_{2}\right)\right)+\sigma_{2}\left(V_{1}\left(x_{1}\right)\right)+\sigma_{r 2}\left(\left|r_{2}\right|\right)
\end{aligned}
$$

hold for all $r \in \mathcal{U}$ along the trajectories $x(t)$ of (1).

This assumption imposes that each subsystem $\Sigma_{i}$ is iISS with respect to input $\left(x_{3-i}, r_{i}\right)$ and state $x_{i}$ (see, for instance [3]). We stress that we have assumed $\alpha_{i} \in \mathcal{K}$ instead of $\alpha_{i} \in \mathcal{P}$ without any loss of generality due to the necessity result in [7] for iISS feedback connections with $\sigma_{i} \in \mathcal{K}$. In the case of cascade, assuming $\alpha_{i} \in \mathcal{K}$ is not necessary [4,5].The following is a result in [10], which is referred to as the iISS smallgain condition in this paper.

Theorem 1. Suppose that Assumption 1 holds and that there exist $\omega_{1}, \omega_{2} \in \mathcal{K}_{\infty}$ satisfying

$$
\begin{aligned}
& \alpha_{1}^{-1} \circ\left(\mathbf{I d}+\omega_{1}\right) \circ \sigma_{1} \circ \alpha_{2}^{-1} \circ\left(\mathbf{I d}+\omega_{2}\right) \circ \sigma_{2}(s) \leq s, \\
& \quad \forall s \in \mathbb{R}_{+} .
\end{aligned}
$$

Then, the following statements hold true:

(i) For $r(t) \equiv 0$, the system (1) is GAS.

(ii) If it holds that

$$
\left\{\lim _{s \rightarrow \infty} \alpha_{i}(s)=\infty \vee \lim _{s \rightarrow \infty} \sigma_{3-i}(s)<\infty\right\}, \quad i=1,2,
$$

then the system (1) is iISS with respect to input $r$ and state $x$.

It is strongly stressed that the small-gain condition (4) with the existence of $\omega_{1}, \omega_{2} \in \mathcal{K}_{\infty}$ implicitly requires that

$$
\lim _{s \rightarrow \infty} \alpha_{2}(s)=\infty \vee \lim _{s \rightarrow \infty} \alpha_{2}(s)>\lim _{s \rightarrow \infty} \sigma_{2}(s) .
$$

It is also important that the property (6) implies that $\Sigma_{2}$ is ISS with respect to its feedback input $x_{1}$ (see for instance [16,3]). On the other hand, $\Sigma_{1}$ does not have to be ISS with respect to its feedback input $x_{2}$. The small-gain condition for iISS subsystems indicates that the interconnection is stable if the stability property of one subsystem, $\Sigma_{2}$, is "strong" enough to compensate the "weak stability" of the other subsystem, $\Sigma_{1}$. Due to this asymmetry, we need to select or interchange the indices "1" and "2" so that (4) holds when iISS subsystems are involved. The condition (4) reduces to the one for the ISS small-gain theorem $[12,11]$ when $\alpha_{1}, \alpha_{2} \in \mathcal{K}_{\infty}$. We have (5) since the nonlinear gain $\alpha_{i}^{-1} \circ\left(\mathbf{I d}+\omega_{i}\right) \circ \sigma_{i}$ of the subsystem $\Sigma_{i}$ is computed independently of the influence of $r_{i}$ from the dissipative inequality (2) or (3). The necessity of the 
condition (6) for stability of the interconnected system is investigated in [10], which is summarized as follows:

Theorem 2. Suppose that $\alpha_{i} \in \mathcal{O}(1)$ and $\sigma_{i} \in \mathcal{O}(>0)$ which are continuously differentiable on $(0, \infty)$ are given for $i=1,2$. Then, the following statements hold true:

(i) The system (1) with $r(t) \equiv 0$ is GAS for all subsystems satisfying Assumption 1 only if

$$
\lim _{s \rightarrow \infty} \alpha_{j}(s) \geq \lim _{s \rightarrow \infty} \sigma_{j}(s)
$$

holds for at least one of $j \in\{1,2\}$.

(ii) The system (1) is ISS with respect to input $r$ and state $x$ for all subsystems satisfying Assumption 1 only if

$$
\lim _{s \rightarrow \infty} \alpha_{j}(s)=\infty \vee \lim _{s \rightarrow \infty} \alpha_{j}(s)>\lim _{s \rightarrow \infty} \sigma_{j}(s)
$$

holds for at least one of $j \in\{1,2\}$.

Renumbering allow us to take $j=2$ for (7) and (8) without any loss of generality. This convention is used in the rest of this paper. The above theorem does not exactly state that (6) is necessary for the iISS of the interconnection. The difference between (6) and (7) is the equality. The main body of this paper does not address the equality case

$$
\infty>\lim _{s \rightarrow \infty} \alpha_{2}(s)=\lim _{s \rightarrow \infty} \sigma_{2}(s)
$$

since it formally prevents us from using the ISS small-gain argument $[12,11]$. Notice that the equality case (9) is incompatible with the small gain condition (4) defined with $\omega_{1}, \omega_{2} \in \mathcal{K}_{\infty}$. Taking into account the necessity of (6) for the ISS case, this paper assumes (6) and makes use of it for proving Theorem 1 in order to interpret the "iISS" small-gain theorem as the combination of "a transient response" and "the ISS small-gain dynamics". In order to give the new interpretation, this paper makes another assumption on the influence of the exogenous signals $r_{1}$ and $r_{2}$, i.e., Assumption 2 , which is the fundamental limitation of the idea of resorting to the "ISS" small-gain argument for "non-ISS" systems.

Remark 1. The necessary conditions in Theorem 2 were proved for $\alpha_{i} \in \mathcal{O}(>1)$ in [10]. It can be verified that $\alpha_{i} \in \mathcal{O}(>1)$ can be replaced by $\alpha_{i} \in \mathcal{O}(1)$ for supply rates given as functions of $V_{1}$ and $V_{2}$ as in Assumption 1 (See [8]).

\section{ESTABLISHING 0-GAS}

This section considers the interconnected system (1) in the absence of the external signals, i.e., $r(t) \equiv 0$, and demonstrates Item (i) of Theorem 1 by means of a transient response plus the ISS small-gain argument. We refer to the stability as 0 -GAS of $\Sigma$. Define the following set:

$$
\mathbf{U}_{2}:=\left\{x_{2} \in \mathbb{R}^{n_{2}}: V_{2}\left(x_{2}\right) \leq \lim _{s \rightarrow \infty} \alpha_{2}^{-1} \circ \sigma_{2}(s)\right\}
$$

Notice that $\mathbf{U}_{2}:=\mathbb{R}^{n_{2}}$ holds if $\lim _{s \rightarrow \infty} \sigma_{2}(s)=\infty$. The following proposition separates each trajectory of the interconnected system $\Sigma$ into two phases.

Proposition 1. Suppose that Assumption 1 holds and that there exist $\omega_{1}, \omega_{2} \in \mathcal{K}_{\infty}$ satisfying (4). Then for each $x_{2}(0) \in \mathbb{R}^{n_{2}}$, there exists $T \in \mathbb{R}_{+}$such that

$$
\begin{aligned}
& x_{2}(t) \in \mathbf{U}_{2}, \quad \forall t \geq T \\
& \sup _{\tau \in[0, T]}|x(\tau)|<\infty .
\end{aligned}
$$

Furthermore, the following two properties hold:

$$
\begin{aligned}
V_{1}\left(x_{1}\right) & \geq \alpha_{1}^{-1} \circ\left(\mathbf{I d}+\omega_{1}\right) \circ \sigma_{1}\left(V_{2}\left(x_{2}\right)\right) \wedge x_{2} \in \mathbf{U}_{2} \\
\Rightarrow \dot{V}_{1} & \leq-\left(\mathbf{I d}-\left(\mathbf{I d}+\omega_{1}\right)^{-1}\right) \circ \alpha_{1}\left(V_{1}\left(x_{1}\right)\right) \\
V_{2}\left(x_{2}\right) & \geq \alpha_{2}^{-1} \circ\left(\mathbf{I d}+\omega_{2}\right) \circ \sigma_{2}\left(V_{1}\left(x_{1}\right)\right) \\
\Rightarrow \dot{V}_{2} & \leq-\left(\mathbf{I d}-\left(\mathbf{I d}+\omega_{2}\right)^{-1}\right) \circ \alpha_{2}\left(V_{2}\left(x_{2}\right)\right) .
\end{aligned}
$$

Proof. Assume that (4) is satisfied for some $\omega_{1}, \omega_{2} \in \mathcal{K}_{\infty}$. It implies that

$$
\begin{aligned}
\sup _{x_{2} \in \mathbf{U}_{2}} \sigma_{1}\left(V_{2}\left(x_{2}\right)\right) & \leq \lim _{s \rightarrow \infty} \sigma_{1} \circ \alpha_{2}^{-1} \circ \sigma_{2}(s) \\
& \leq \lim _{s \rightarrow \infty}\left(\mathbf{I d}+\omega_{1}\right)^{-1} \circ \alpha_{1}(s) .
\end{aligned}
$$

From this property and (2)-(3) it follows that the properties (12) and (13) hold. Next, suppose for the time being that

$$
\lim _{s \rightarrow \infty} \alpha_{1}(s)<\infty \wedge \lim _{s \rightarrow \infty} \alpha_{1}(s) \leq \lim _{s \rightarrow \infty} \sigma_{1}(s) .
$$

Then, the small-gain condition (4) implies that there exists a positive constant $\sigma_{2}^{\max }$ such that

$$
\lim _{s \rightarrow \infty} \sigma_{2}(s) \leq \sigma_{2}^{\max }<\infty .
$$

Since $\sigma_{2}^{\max }$ is independent of $x_{1}$, the dissipation inequality (3) of $\Sigma_{2}$ and the property (6) implied by (4) guarantee that the state $x_{2}(t)$ is bounded and eventually enters the forward invariant set $\mathbf{U}_{2}$, i.e., (10). In fact, there exists $\delta>0$ such that $\dot{V}_{2} \leq-\delta$ holds for all $x_{2} \notin \mathbf{U}_{2}$. Note that $T \geq 0$ fulfilling (10) is finite, and that the state $x_{1}(t)$ is bounded over the time interval $[0, T]$ since $\Sigma_{1}$ is iISS with respect to input $x_{2}$. Thus, we have (11). Finally, in the case that (14) does not hold, that is:

$$
\lim _{s \rightarrow \infty} \alpha_{1}(s)=\infty \vee \lim _{s \rightarrow \infty} \alpha_{1}(s)>\lim _{s \rightarrow \infty} \sigma_{1}(s),
$$

the subsystem $\Sigma_{1}$ is ISS with respect input $x_{2}$, and we can invoke the ISS small-gain argument $[12,11]$ to obtain (10) and 
(11). The time $T$ can be made independent of $x_{1}(0)$ since $\mathbf{U}_{2} \neq \mathbb{R}^{n_{2}}$ implies (15).

The property (12) implies that $\Sigma_{1}$ exhibits an ISS property when the input $x_{2}$ is restricted to $\mathbf{U}_{2}$. Note that $\quad \mathbf{I d}-\left(\mathbf{I d}+\omega_{i}\right)^{-1} \in \mathcal{K}_{\infty}$ since $\quad\left(\mathbf{I d}-\left(\mathbf{I d}+\omega_{i}\right)^{-1}\right) \circ(s+$ $\left.\omega_{i}(s)\right)=\omega_{i}(s)$. Due to (12) and (13), the convergence of $x(t)$ to the origin $x=0$ departing from any $x(T) \in \mathbb{R}^{n_{1}} \times \mathbf{U}_{2}$ at $t=T$ is ensured by the small-gain condition (4). For instance, we can follow the proof for the interconnection of the two ISS subsystems given in [12,11] and [17] which describes the small-gain argument with the domain restriction. Therefore, Proposition 1 yields a proof of Item (i) of Theorem 1. The behavior before $t=T$ described by (10) and (11) is transient. After the finite time $t=T$, the contractive dynamics kicks in since the iISS small-gain condition acts as the ISS smallgain condition in the domain $\mathbf{U}_{2}$ where the trajectories evolve.

Remark 2. In the absence of external signals, i.e. $r(t) \equiv 0$, a Lyapunov function establishing the GAS of the interconnected system can be constructed even when (9) holds. In fact, Theorem 1 in [10] derives such a Lyapunov function from a small-gain condition. The small-gain condition is exactly in the form of (4). However, the amplification factors $\omega_{1}, \omega_{2}$ in (4) for GAS are not necessarily of class $\mathcal{K}_{\infty}$ in the absence of the exogenous signal $r$. Although the above argument in this section does not explicitly address (9), the observation of the transient plus the ISS small-gain dynamics still holds true. Notice that the ISS small-gain theorem $[12,11]$ applies to the case of

$$
\lim _{s \rightarrow \infty} \alpha_{1}(s)=\lim _{s \rightarrow \infty} \sigma_{1}(s) \wedge \text { Eq. }(9)
$$

directly since $V_{1}$ and $V_{2}$ become ISS Lyapunov functions of the individual subsystems. Therefore, Proposition 1 holds true even for $\omega_{i} \notin \mathcal{K}_{\infty}$ in the case of (16). If

$$
\lim _{s \rightarrow \infty} \alpha_{1}(s)>\lim _{s \rightarrow \infty} \sigma_{1}(s) \wedge \text { Eq. (9) }
$$

holds, by virtue of $\alpha_{2}^{-1} \circ \sigma_{2} \in K_{\infty}$, the argument given in this section can be used by switching the indices "1" and " 2 ". Note that the property (17) with the switching allows us to assume $\omega_{1}, \omega_{2} \in \mathcal{K}_{\infty}$ for (4) there. The situation

$$
\lim _{s \rightarrow \infty} \alpha_{1}(s)<\lim _{s \rightarrow \infty} \sigma_{1}(s) \wedge \text { Eq. }(9)
$$

is excluded by theorem 5 (i) in [10]. Therefore, for the GAS case (i.e., for $r(t) \equiv 0$ ), the interpretation of the transient plus the ISS small-gain dynamics is valid whenever

$$
\lim _{s \rightarrow \infty} \alpha_{2}(s) \geq \lim _{s \rightarrow \infty} \sigma_{2}(s)
$$

\section{ESTABLISHING IISS}

This section proves Item (ii) of Theorem 1 under an assumption about disturbance magnitude. The property (6) implied by the small-gain condition (4) again plays a key role in implementing the idea of a transient plus the ISS smallgain argument. The proof consists of two parts. One is to verify that the system (1) is 0-GAS (that is, GAS when $r(t) \equiv 0)$. The other part is to establish the Bounded Energy Frequently Bounded State (BEFBS) property of the system (1). It is shown in [2] that the combination of the above two properties is equivalent to the iISS property of the system (1). Since the 0 -GAS has been proved in the previous section, this section is devoted to the BEFBS property.

First, notice that $\lim _{s \rightarrow \infty} \alpha_{i}(s)>\lim _{s \rightarrow \infty} \sigma_{i}(s)$ does not guarantee the ISS property of $\Sigma_{i}$ with respect to input $\left(x_{3-i}, r_{i}\right)$ since $\lim _{s \rightarrow \infty} \sigma_{r i}(s)$ can anyway be larger than $\lim _{s \rightarrow \infty} \alpha_{i}(s)$. In fact, when there exists $i \in\{1,2\}$ such that $\lim _{s \rightarrow \infty} \alpha_{i}(s)<\infty$ holds, the previously existing results show only the iISS of the interconnected system $[6,10]$. Hence, in contrast to the GAS case, the condition $\lim _{s \rightarrow \infty} \alpha_{2}(s)>\lim _{s \rightarrow \infty} \sigma_{2}(s)$ is not sufficient for resorting to the ISS small-gain argument in the presence of external inputs. Therefore, in order to make use of the small-gain argument of ISS-type, we introduce the following:

Assumption 2. The following properties hold:

$$
\begin{aligned}
& \lim _{s \rightarrow \infty} \alpha_{2}(s)=\infty \vee \lim _{s \rightarrow \infty} \alpha_{2}(s)>\lim _{s \rightarrow \infty}\left(\mathbf{I d}+\omega_{2}\right) \circ \sigma_{2}(s) \\
& \lim _{s \rightarrow \infty} \omega_{1} \circ \sigma_{1} \circ \alpha_{2}^{-1} \circ\left(\mathbf{I d}+\omega_{2}\right) \circ \sigma_{2}(s) \\
& \geq \lim _{s \rightarrow \infty}\left\{\sigma_{1} \circ \alpha_{2}^{-1} \circ\left(\mathbf{I d}+\omega_{2}^{-1}\right) \circ \sigma_{r 2}(s)+\sigma_{r 1}(s)\right\} \\
& \lim _{s \rightarrow \infty} \alpha_{2}(s)=\infty \vee \\
& \lim _{s \rightarrow \infty} \alpha_{2}(s)>\lim _{s \rightarrow \infty}\left(\mathbf{I d}-\left(\mathbf{I d}+\omega_{2}\right)^{-1}\right)^{-1} \circ \sigma_{r 2}(s) .
\end{aligned}
$$

Notice that we can pick $\omega_{2} \in \mathcal{K}_{\infty}$ fulfilling (20) whenever there exists a pair of $\omega_{1}, \omega_{2} \in \mathcal{K}_{\infty}$ satisfying (4). Define:

$$
\mathbf{U}_{D 2}:=\left\{x_{2} \in \mathbb{R}^{n_{2}}: V_{2}\left(x_{2}\right) \leq \lim _{s \rightarrow \infty} \alpha_{2}^{-1} \circ\left\{\sigma_{2}(s)+\sigma_{r_{2}}(s)\right\}\right\} .
$$

Let $\mathbf{U}_{D 2}:=\mathbb{R}^{n_{2}}$ if $\lim _{s \rightarrow \infty} \alpha_{2}(s)<\lim _{s \rightarrow \infty} \sigma_{2}(s)+\sigma_{r 2}(s)$. The next proposition shows that the above assumption allows us to separate each trajectory of the interconnected system $\Sigma$ into two phases even in the presence of the external inputs.

Proposition 2. Suppose that Assumption 1 and the property (5) hold. Assume that there exist $\omega_{1}, \omega_{2} \in \mathcal{K}_{\infty}$ satisfying (4) and Assumption 2. Then for each $x_{2}(0) \in \mathbb{R}^{n_{2}}$, there exists $T \in \mathbb{R}_{+}$such that 


$$
\begin{aligned}
& x_{2}(t) \in \mathbf{U}_{D 2}, \quad \forall t \geq T \\
& \sup _{\tau \in[0, T]}|x(\tau)|<\infty \\
& \mathbf{U}_{D 2}=\mathbb{R}^{n_{2}} \Rightarrow T=0
\end{aligned}
$$

hold for any measurable, locally essentially bounded $r$. Furthermore, there exist $\gamma \in \mathcal{K}_{\infty}$ and $w \in \mathbb{R}_{+}$such that

$$
\begin{gathered}
\sup _{t \in[T, \infty)}|r(t)| \leq l \Rightarrow \underset{t \rightarrow \infty}{\limsup }|x(t)| \leq \gamma(l)+w \\
\forall x_{1}(T) \in \mathbb{R}^{n_{1}}, \quad x_{2}(T) \in \mathbf{U}_{D 2} .
\end{gathered}
$$

Proof. First, assume that

$$
\lim _{s \rightarrow \infty} \sigma_{1}(s)=\lim _{s \rightarrow \infty} \sigma_{2}(s)=\infty
$$

is satisfied. Then the condition (4) and the implicit requirement (6) yield $\lim _{s \rightarrow \infty} \alpha_{1}(s)=\lim _{s \rightarrow \infty} \alpha_{2}(s)=\infty$ and $\mathbf{U}_{2}=\mathbb{R}^{n_{2}}$. We obtain (23), (24) and (26) for any $T \in \mathbb{R}_{+}$by using the ISS small-gain result in $[12,11]$. Hence, in the rest of the proof, we assume

$$
\lim _{s \rightarrow \infty} \sigma_{1}(s)<\infty \vee \lim _{s \rightarrow \infty} \sigma_{2}(s)<\infty .
$$

The definition of $x_{2} \in \mathbf{U}_{D 2}$ yields

$$
\begin{aligned}
\sup _{x_{2} \in \mathbf{U}_{D 2}} \sigma_{1}\left(V_{2}\left(x_{2}\right)\right) \leq & \lim _{s \rightarrow \infty} \sigma_{1} \circ \alpha_{2}^{-1} \circ\left\{\sigma_{2}(s)+\sigma_{r 2}(s)\right\} \\
\leq & \lim _{s \rightarrow \infty} \sigma_{1} \circ \alpha_{2}^{-1} \circ\left(\mathbf{I d}+\omega_{2}\right) \circ \sigma_{2}(s) \\
& +\lim _{s \rightarrow \infty} \sigma_{1} \circ \alpha_{2}^{-1} \circ\left(\mathbf{I d}+\omega_{2}^{-1}\right) \circ \sigma_{r 2}(s)
\end{aligned}
$$

To derive the second inequality, the two cases separated by $\omega_{2} \circ \sigma_{2}(s) \geq \sigma_{r 2}(s)$ and $\omega_{2} \circ \sigma_{2}(s)<\sigma_{r 2}(s)$ are combined. From (2) it follows that, for all $x_{2} \in \mathbf{U}_{D 2}$,

$$
\begin{aligned}
\dot{V}_{1} \leq & -\alpha_{1}\left(V_{1}\right)+\lim _{s \rightarrow \infty} \sigma_{1} \circ \alpha_{2}^{-1} \circ\left(\mathbf{I d}+\omega_{2}\right) \circ \sigma_{2}(s) \\
& +\lim _{s \rightarrow \infty} \sigma_{1} \circ \alpha_{2}^{-1} \circ\left(\mathbf{I d}+\omega_{2}^{-1}\right) \circ \sigma_{r 2}(s)+\sigma_{r 1}\left(\left|r_{1}\right|\right) .
\end{aligned}
$$

The property (21) applied to the above gives

$$
\dot{V}_{1} \leq-\alpha_{1}\left(V_{1}\right)+\lim _{s \rightarrow \infty}\left(\mathbf{I d}+\omega_{1}\right) \circ \sigma_{1} \circ \alpha_{2}^{-1} \circ\left(\mathbf{I d}+\omega_{2}\right) \circ \sigma_{2}(s)
$$

Recall that (20) and (28) imply

$$
\lim _{s \rightarrow \infty}\left(\mathbf{I d}+\omega_{1}\right) \circ \sigma_{1} \circ \alpha_{2}^{-1} \circ\left(\mathbf{I d}+\omega_{2}\right) \circ \sigma_{2}(s)<\infty .
$$

Applying this property and (4) to (30) and (29), we can verify that there exist a function $\gamma_{1} \in \mathcal{K}_{\infty}$ and a constant $w_{1} \geq 0$ such that

$$
\begin{aligned}
& \sup _{t \in[T, \infty)}\left|r_{1}(t)\right| \leq l_{1} \Rightarrow\left\{\begin{array}{l}
\sup _{t \in[T, \infty)}\left|x_{1}(t)\right|<\infty \\
\limsup _{t \rightarrow \infty}\left|x_{1}(t)\right| \leq \gamma_{1}\left(l_{1}\right)+w_{1}
\end{array}\right. \\
& \forall x_{1}(T) \in \mathbb{R}^{n_{1}}, \quad x_{2}(T) \in \mathbf{U}_{D 2} .
\end{aligned}
$$

If

$$
\lim _{s \rightarrow \infty} \alpha_{2}(s)>\lim _{s \rightarrow \infty} \sigma_{2}(s)+\sigma_{r 2}(s),
$$

is satisfied, the boundedness of $\mathbf{U}_{D 2}$ and (31) yield the bounded-input bounded-state property (26) over $t \in[T, \infty)$ provided that (23) holds. In the case of

$$
\lim _{s \rightarrow \infty} \alpha_{2}(s)=\lim _{s \rightarrow \infty} \sigma_{2}(s)+\sigma_{r 2}(s)=\infty,
$$

the set $\mathbf{U}_{D 2}$ is unbounded, i.e., $\mathbf{U}_{D 2}=\mathbb{R}^{n_{2}}$. We temporarily assume that

$$
\lim _{s \rightarrow \infty} \alpha_{1}(s)<\infty \vee \lim _{s \rightarrow \infty} \alpha_{2}(s)<\infty .
$$

This property ensures $\lim _{s \rightarrow \infty} \sigma_{2}(s)<\infty$. Indeed, it is implied by (5) if $\lim _{s \rightarrow \infty} \alpha_{1}(s)<\infty$. The property (6) yields $\lim _{s \rightarrow \infty} \sigma_{2}(s)<\infty$ in the case of $\lim _{s \rightarrow \infty} \alpha_{2}(s)<\infty$. The dissipation inequality (3) of $\Sigma_{2}$ with $\lim _{s \rightarrow \infty} \sigma_{2}(s)<\infty$ guarantees the existence of a function $\gamma_{2} \in \mathcal{K}_{\infty}$ and a constant $w_{2} \geq 0$ such that

$$
\begin{gathered}
\sup _{t \in[T, \infty)}\left|r_{2}(t)\right| \leq l_{2} \Rightarrow\left\{\begin{array}{l}
\sup _{t \in[T, \infty)}\left|x_{2}(t)\right|<\infty \\
\limsup \left|x_{2}(t)\right| \leq \gamma_{2}\left(l_{2}\right)+w_{2}
\end{array}\right. \\
\forall x_{1}(T) \in \mathbb{R}^{n_{1}}, \quad x_{2}(T) \in \mathbf{U}_{D 2}=\mathbb{R}^{n_{2}} .
\end{gathered}
$$

Combining (31) and (35) yields (26) for an arbitrary $T \in \mathbb{R}_{+}$. We now retract (34) and assume that

$$
\lim _{s \rightarrow \infty} \alpha_{1}(s)=\infty \wedge \lim _{s \rightarrow \infty} \alpha_{2}(s)=\infty
$$

holds. Then the standard ISS small-gain theorem yields (23), (24) and (26) with $T=0$ since $\mathbf{U}_{D 2}=\mathbb{R}^{n_{2}}$.

To see that one of (32) and (33) must hold, consider (22). Then there exists $\beta \in \mathcal{K}_{\infty}$ such that

$$
\begin{aligned}
& \lim _{s \rightarrow \infty} \alpha_{2}(s)=\infty \vee \\
& \lim _{s \rightarrow \infty} \alpha_{2}(s) \geq \lim _{s \rightarrow \infty}\left((\mathbf{I d}+\beta)^{-1}-\left(\mathbf{I d}+\omega_{2}\right)^{-1}\right)^{-1} \circ \sigma_{r 2}(s) .
\end{aligned}
$$

By virtue of (37) and

$$
\lim _{s \rightarrow \infty} \alpha_{2}(s) \geq \lim _{s \rightarrow \infty}\left(\mathbf{I d}+\omega_{2}\right) \circ \sigma_{2}(s)
$$

implied by (4), the property

$$
\lim _{s \rightarrow \infty} \alpha_{2}(s) \geq \lim _{s \rightarrow \infty}(\mathbf{I d}+\beta) \circ\left(\sigma_{2}(s)+\sigma_{r 2}(s)\right)
$$

holds since 


$$
\begin{gathered}
\lim _{s \rightarrow \infty} \sigma_{2}(s)+\lim _{s \rightarrow \infty} \sigma_{r 2}(s) \leq \lim _{s \rightarrow \infty}\left(\left(\mathbf{I d}+\omega_{2}\right)^{-1}\right. \\
\left.+(\mathbf{I d}+\beta)^{-1}-\left(\mathbf{I d}+\omega_{2}\right)^{-1}\right) \circ \alpha_{2}(s)
\end{gathered}
$$

is satisfied for $\lim _{s \rightarrow \infty} \alpha_{2}(s)<\infty$. Thus, we arrive at one of (32) and (33).

Next, we shall establish (23), (24) and (25). Since the case where both (33) and (36) hold has already been solved, we only need to consider the complementary case. If (33) and (34) hold, we have obtained $\mathbf{U}_{D 2}=\mathbb{R}^{n_{2}}$ for which (31), (35) and (26) are satisfied for an arbitrary $T \in \mathbb{R}_{\text {+. Taking } T=0 \text { is }}$ satisfactory for (23), (24) and (25). Consider the remaining case where (32) holds. By virtue of $\mathbf{U}_{D 2} \neq \mathbb{R}^{n_{2}}$, the implication (25) holds true, i.e., it can be skipped. The dissipation inequality (3) of $\Sigma_{2}$ guarantees that the state $x_{2}(t)$ which is bounded enters the set $\mathbf{U}_{D 2}$ in a finite time $T \geq 0$ and remains there, i.e., (23), and $T$ can be picked independently of $x_{1}(0)$. Note that the state $x_{1}(t)$ is also bounded in the time interval $[0, T]$ since $\Sigma_{1}$ is iISS with respect to input $\left(x_{2}, r_{1}\right)$ and state $x_{1}$. Hence, we arrive at (24).

Proposition 2 demonstrates that, even in the presence of the external signal $r$, the behavior up to $t=T$ can be considered as a transient in view of (23) and (24). After $t=T$, the bounded-input bounded-state property (26) takes effect since the iISS small-gain condition acts as the ISS small-gain condition in the domain $\mathbf{U}_{D 2}$ where the trajectories evolve. The bounded-input bounded-state property for $t \in[T, \infty)$ preceded by the transient for $t \in[0, T)$ implies the BEFBS property of $\Sigma$ with respect to input $r$ and state $x$. Note that we have $T=0$ if $\mathbf{U}_{D 2}=\mathbb{R}^{n_{2}}$. These facts together with the 0 -GAS proved in the previous section complete the proof of Item (ii) of Theorem 1.

\section{ANOTHER FORMULATION FOR DISTURBANCE}

In the presence of external signals, the idea of the reduction to the ISS small-gain argument in the presence of an iISS subsystem can be seen more or less in a compact manner if one uses dissipation inequalities of another type for the iISS property of the individual subsystems. To this end, in this section, we replace Assumptions 1 and 2 with the following two assumptions.

Assumption 3. For each $i \in\{1,2\}$, there exist a continuously differentiable positive definite and radially unbounded function $V_{i}: \mathbb{R}^{n_{i}} \rightarrow \mathbb{R}_{+}$and class $\mathcal{K}$ functions $\alpha_{i}, \sigma_{i}, \sigma_{r i}$ such that

$$
\begin{aligned}
& \dot{V}_{1} \leq-\alpha_{1}\left(V_{1}\left(x_{1}\right)\right)+\max \left\{\sigma_{1}\left(V_{2}\left(x_{2}\right)\right), \sigma_{r 1}\left(\left|r_{1}\right|\right)\right\} \\
& \dot{V}_{2} \leq-\alpha_{2}\left(V_{2}\left(x_{2}\right)\right)+\max \left\{\sigma_{2}\left(V_{1}\left(x_{1}\right)\right), \sigma_{r 2}\left(\left|r_{2}\right|\right)\right\}
\end{aligned}
$$

hold for all $r \in \mathcal{U}$ along the trajectories $x(t)$ of (1).

Assumption 4. The following properties hold:

$$
\begin{aligned}
& \lim _{s \rightarrow \infty} \sigma_{1} \circ \alpha_{2}^{-1} \circ \sigma_{2}(s) \geq \max \lim _{s \rightarrow \infty}\left\{\sigma_{1} \circ \alpha_{2}^{-1} \circ \sigma_{r 2}(s), \sigma_{r 1}(s)\right\} \\
& \lim _{s \rightarrow \infty} \alpha_{2}(s)=\infty \vee \lim _{s \rightarrow \infty} \alpha_{2}(s)>\lim _{s \rightarrow \infty} \sigma_{r 2}(s) .
\end{aligned}
$$

Assumption 3 is quantitatively different from Assumption 1 . However, they are qualitatively equivalent in view of the standard relation $a+b \leq \max \{2 a, 2 b\} \leq 2 a+2 b$ for $a, b \in \mathbb{R}_{+}$. The quantitative difference in the formulation of subsystems brings in the technical difference between Assumptions 2 and 4. Indeed, when the interconnection of two iISS subsystems is defined with Assumption 3 in Theorem 1, we are able to achieve the reduction to the transient plus the ISS small-gain argument under Assumption 4 which may look more intuitive than Assumption 2. The rest of this section sketches this fact.

Since the 0-GAS property is proved in Section III, we shall prove the BEFBS property of the system (1). As in Section IV, we can assume (34). The properties (5) and (6) ensure $\lim _{s \rightarrow \infty} \sigma_{2}(s)<\infty$. Suppose that $\lim _{s \rightarrow \infty} \sigma_{r 2}(s)<\infty$. Due to (42) and (6), the dissipation inequality (40) of $\Sigma_{2}$ guarantees that the state $x_{2}(t)$ which is bounded enters the set

$$
\mathbf{U}_{M 2}:=\left\{x_{2} \in \mathbb{R}^{n_{2}}: V_{2}\left(x_{2}\right) \leq \lim _{s \rightarrow \infty} \alpha_{2}^{-1} \circ \max \left\{\sigma_{2}(s), \sigma_{r 2}(s)\right\}\right\}
$$

in a finite time $T$ and stays there. When $\lim _{s \rightarrow \infty} \sigma_{r 2}(s)=\infty$ holds, the same property holds with $T$ which satisfies $T<\infty$ for each $\sup _{t \in[0, \infty)}\left|r_{2}(t)\right|<\infty$. The state $x_{1}(t)$ is also bounded for the time interval $[0, T]$ since $\Sigma_{1}$ is iISS with respect to input $\left(x_{2}, r_{1}\right)$ and state $x_{1}$. By definition we have:

$$
\begin{aligned}
& \sup _{x_{2} \in \mathbf{U}_{M 2}} \sigma_{1}\left(V_{2}\left(x_{2}\right)\right) \leq \\
& \quad \max \left\{\lim _{s \rightarrow \infty} \sigma_{1} \circ \alpha_{2}^{-1} \circ \sigma_{2}(s), \lim _{s \rightarrow \infty} \sigma_{1} \circ \alpha_{2}^{-1} \circ \sigma_{r 2}(s)\right\} .
\end{aligned}
$$

From (39) it follows that, for all $x_{2} \in \mathbf{U}_{M 2}$,

$$
\begin{gathered}
\dot{V}_{1} \leq-\alpha_{1}\left(V_{1}\left(x_{1}\right)\right)+\max \left\{\lim _{s \rightarrow \infty} \sigma_{1} \circ \alpha_{2}^{-1} \circ \sigma_{2}(s),\right. \\
\left.\lim _{s \rightarrow \infty} \sigma_{1} \circ \alpha_{2}^{-1} \circ \sigma_{r 2}(s), \sigma_{r 1}\left(\left|r_{1}\right|\right)\right\} .
\end{gathered}
$$

Here, the property $\lim _{s \rightarrow \infty} \sigma_{1} \circ \alpha_{2}^{-1} \circ \sigma_{2}(s)<\infty$ holds due to $\lim _{s \rightarrow \infty} \sigma_{2}(s)<\infty$ and (6). Thus, the assumption (41) and the small-gain condition (4) lead us to the bounded-input bounded-state property (31) with respect to state $x_{1}$ in the interval of $t \in[T, \infty)$ for the initial conditions $x_{2}(T) \in \mathbf{U}_{M 2}$. These facts allow us to arrive at Proposition 2 replacing Assumptions 1 and 2 with Assumptions 3 and 4. Hence, we obtain the BEFBS property of $\Sigma$, and Item (ii) of Theorem 1 is proved. 
Assumption 1 and Assumption 3 are qualitatively equivalent in the sense that $\sigma_{i}+\sigma_{r i} \leq \max \left\{2 \sigma_{i}, 2 \sigma_{r i}\right\} \leq$ $2 \sigma_{i}+2 \sigma_{r i}$. We can consider other variants of dissipation inequalities for iISS. Although the coefficients appearing in the transformation between two representations result in conservativeness in different forms, the essence of imposing the constraint on the external inputs for the reduction to the ISS small-gain argument remains the same.

Remark 3. As already stressed, the difficulty in establishing the iISS via the transient plus the ISS small-gain dynamics arises when the effect of $r_{i}$ 's is larger than the contribution of $\alpha_{i}$ 's. Both Assumption 1 and Assumption 3 allow the magnitude of $\sigma_{r i}$ 's to be arbitrarily large. In order to make the ISS small-gain argument work, the undesirably large effect of $r_{i} \mathrm{~s}$ is avoided by Assumptions 2 and 4. In short, $\sigma_{r i} \mathrm{~s}$ are required to be sufficiently small in this paper. It is worth noting that the pair of ISS with respect to small inputs and forward completeness does not always imply iISS. Indeed, one can construct a forward complete non-iISS system which is ISS with respect to small inputs by modifying the technique proposed in [3, Section V]. In the presence of arbitrarily large $\sigma_{r i}$ 's, removing Assumptions 2 and 4 is inherently difficult.

Remark 4. Neither the pair (21)-(22) nor the pair (41)(42) is necessary for establishing the iISS property of the interconnection of iISS subsystems. For example, in the case where $\alpha_{1}=\sigma_{2}, \alpha_{2}=d \sigma_{1}$ with $\sigma_{r 1}, \sigma_{r 2} \in \mathcal{K}_{\infty}$ and some $d>1$, the function $V=V_{1}+V_{2}(1+1 / d) / 2$ is an iISS Lyapunov function, thus immediately proving the iISS of the interconnection. In contrast to the approach pursued in this paper, this case is covered by the iISS small-gain theorems proposed in $[6,10]$. Therefore, the approach based on the ISS small-gain argument plus the transient is more restrictive than the direct iISS small-gain approach.

\section{AN ILLUSTRATIVE EXAMPLE}

Consider the interconnected system described by:

$$
\begin{aligned}
& \dot{x}_{1}=-\frac{x_{1}}{1+x_{1}^{2}}+\frac{x_{1}}{2\left(1+x_{1}^{2}\right)}\left(x_{2}+r_{1}\right) \\
& \dot{x}_{2}=-x_{2}+\frac{x_{1}^{2}}{1+x_{1}^{2}}
\end{aligned}
$$

This pair satisfies the dissipation inequalities

$$
\dot{V}_{1} \leq-\frac{2 V_{1}\left(x_{1}\right)}{1+V_{1}\left(x_{1}\right)}+\sqrt{V_{2}\left(x_{2}\right)}+\left|r_{1}\right|
$$

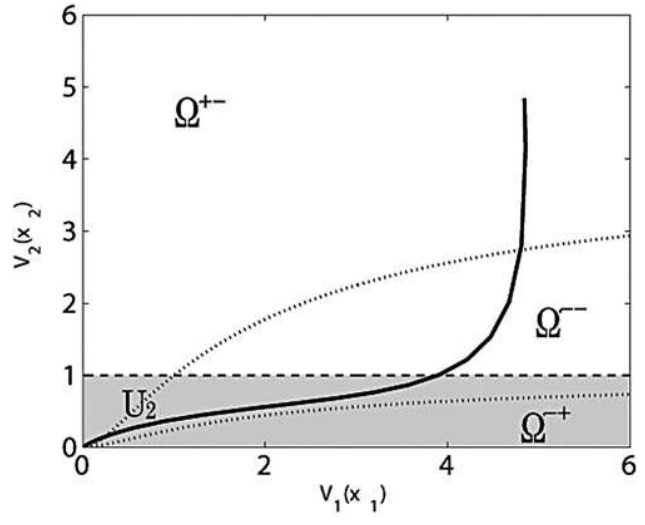

Fig. 1. The trajectory of (44)-(45) on the $\left(V_{1}, V_{2}\right)$-plane for $r_{1}(t) \equiv 0$ and $x(0)=[2.2,2.2]^{T}$ with relation to the sets $\Omega^{+-}, \Omega^{-}, \Omega^{-+}$and $\mathbf{U}_{2}$.

$$
\dot{V}_{2} \leq-V_{2}\left(x_{2}\right)+\left(\frac{V_{1}\left(x_{1}\right)}{1+V_{1}\left(x_{1}\right)}\right)^{2}
$$

for $V_{1}\left(x_{1}\right)=x_{1}^{2}$ and $V_{2}\left(x_{2}\right)=x_{2}^{2}$. Note that the upper bounds in (46) and (47), i.e., the supply rates, may not be completely tight. The subsystem $\Sigma_{1}$ is not ISS with respect to input $x_{2}$, and it is only iISS. The trajectory of (44)-(45) for the initial condition $x(0)=[2.2,2.2]^{T}$ is plotted on the $\left(V_{1}, V_{2}\right)$-plane in Fig. 1 for $r_{1}(t) \equiv 0$. Fig. 1 also depicts the following sets:

$$
\begin{aligned}
& \Omega^{+-}:=\left\{\left(V_{1}, V_{2}\right) \in \mathbb{R}_{+}^{2}: \alpha_{1}\left(V_{1}\right) \leq \sigma_{1}\left(V_{2}\right) \wedge \alpha_{2}\left(V_{2}\right) \geq \sigma_{2}\left(V_{1}\right)\right\} \\
& \Omega^{--}:=\left\{\left(V_{1}, V_{2}\right) \in \mathbb{R}_{+}^{2}: \alpha_{1}\left(V_{1}\right) \geq \sigma_{1}\left(V_{2}\right) \wedge \alpha_{2}\left(V_{2}\right) \geq \sigma_{2}\left(V_{1}\right)\right\} \\
& \Omega^{-+}:=\left\{\left(V_{1}, V_{2}\right) \in \mathbb{R}_{+}^{2}: \alpha_{1}\left(V_{1}\right) \geq \sigma_{1}\left(V_{2}\right) \wedge \alpha_{2}\left(V_{2}\right) \leq \sigma_{2}\left(V_{1}\right)\right\} .
\end{aligned}
$$

The boundaries of these sets are not necessarily the nullclines of (44) and (45) owing to the lack of tightness in the dissipation inequalities (46) and (47). Two phases are observed in Fig. 1. The first phase is the transient evolving outside $\mathbf{U}_{2}$ for which the trajectory heads. The second phase is the trajectory converging to the origin without leaving $\mathbf{U}_{2}$. Once the trajectory enters the positively invariant set $\mathbf{U}_{2}$, the dynamic is governed by the ISS small-gain condition as discussed in Section III. It is also seen in Fig. 1 near the origin that the set $\Omega^{--}$is too narrow to be an invariant set because of the gaps in the dissipation inequalities. Fig. 2 shows the response for the same initial condition in the presence of the disturbance $r_{1}(t)=1.8 /(2+t)$. The trajectory is bounded and moves toward the set $\mathbf{U}_{2}$ which becomes positively invariant again. Since the iISS small-gain condition acts as the ISS small-gain condition in $\mathbf{U}_{2}$, we see that the trajectory converges to the origin. It conforms to the converging-input converging-state of the ISS property. The boundedness and the converging property agree with the iISS property for the entire $t \geq 0$ which is established in Section IV. 


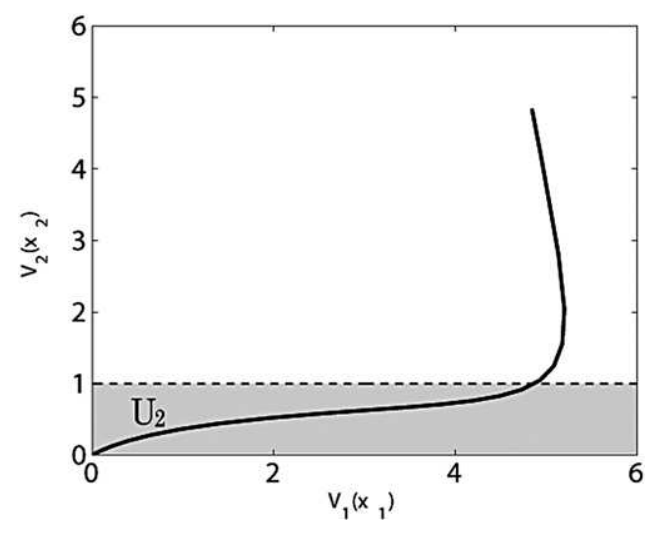

Fig. 2. The trajectory of (44)-(45) on the $\left(V_{1}, V_{2}\right)$-plane for $r_{1}(t)=1.8 /(2+t)$ and $x(0)=[2.2,2.2]^{T}$ with relation to the set $\mathbf{U}_{2}$.

\section{CONCLUDING REMARKS}

The iISS small-gain theorem developed in $[6,10]$ for interconnections of two iISS subsystems has been revisited to give it a trajectory-based interpretation linking with the contractive mechanism of the ISS small-gain theorem. According to the preceding study, an interconnection involving a nonISS subsystem is stable only if the other subsystem is ISS with respect to its feedback input. By making use of this fact, this paper has shown that the behavior of the interconnected system can be split into two phases. In the first phase, roughly, the trajectory of the ISS subsystem behaves almost independently of the other iISS subsystem and this phase lasts until the trajectory of the ISS subsystem enters a neighborhood $\mathbf{U}_{2}$ of the origin with a certain radius. In this phase, the behavior of the merely iISS subsystem is almost a free response. In the second phase, the interaction between the two subsystems takes effect and the contractive behavior of the whole state vector occurs since the small-gain constraint plays the role of the ISS small-gain condition in $\mathbf{U}_{2}$. This observation would be practically useful in designing and analyzing the dynamics of nonlinear control systems based on the iISS small-gain theorem. It is worth stressing that the above interpretation is not always applicable. The external signals are not allowed to be large either, as in (21)-(22) or (41)-(42). These assumptions ensure that the transient response actually dies in finite time which allows us to make use of the "ISS" small-gain argument for the subsequent behavior in dealing with "iISS" subsystems. There are interconnected systems which violate these assumptions and can anyway be proved to be iISS with respect to the external signals by constructing Lyapunov functions as in $[6,10]$.

The independent study in [13] reported very recently also combines the transient with a small-gain argument for a system class which overlaps with the class of systems this paper deals with although the study [13] does not formulate systems in the framework of iISS. In [13], another stability property so-called input-to-output stability is verified by computing the input-to-output gain of interconnected systems under the assumption that an estimate of trajectories is somehow available during a finite time period when an ISStype small-gain criterion is invalid. External signals can be incorporated into the stability analysis as far as the above assumption is fulfilled. In contrast, this paper does not assume anything more than the standard iISS dissipation inequalities of subsystems, which would be less demanding than the time embedded trajectory estimate used in [13]. An abstract model in [13] covers a considerably broad class of systems at the price of some complexities in the stability criterion.

\section{REFERENCES}

1. Angeli, D. and A. Astolfi, "A tight small gain theorem for not necessarily ISS systems," Syst. Control Lett., Vol. 56, pp. 87-91 (2007).

2. Angeli, D., B. Ingalls, E. D. Sontag and Y. Wang, "Separation principles for input-output and integral-input-tostate stability," SIAM J. Control Optim., Vol. 43, pp. 256-276 (2004).

3. Angeli, D., E. D. Sontag and Y. Wang, "A characterization of integral input-to-state stability," IEEE Trans. Autom. Control, Vol. 45, pp. 1082-1097 (2000).

4. Arcak, M., D. Angeli, and E. Sontag, "A unifying integral ISS framework for stability of nonlinear cascades," SIAM J. Control Optim., Vol. 40, pp. 1888-1904 (2002).

5. Chaillet, A. and D. Angeli, "Integral input to state stable systems in cascade," Syst. Control Lett., Vol. 57, pp. 519-527 (2008).

6. Ito, H., "State-dependent scaling problems and stability of interconnected iISS and ISS systems," IEEE Trans. Autom. Control, Vol. 51, pp. 1626-1643 (2006).

7. H. Ito, "A Lyapunov approach to cascade interconnection of integral input-to-state stable systems," IEEE Trans. Autom. Control, Vol. 55, pp. 702-708 (2010).

8. Ito, H., "Necessary conditions for stability of networks of iISS systems," Proc. 49th IEEE Conf. Decision Contr., pp. 7401-7406 (2010).

9. Ito, H., R. A. Freeman, and A. Chaillet. "Interpreting the iISS small-gain theorem as transient plus ISS small-gain regulation," Proc. 49th IEEE Conf. Decision Contr., pp. 2432-2437 (2010).

10. Ito, H. and Z. P. Jiang, "Necessary and sufficient small gain conditions for integral input-to-state stable systems: A Lyapunov perspective," IEEE Trans. Autom. Control, Vol.54, pp. 2389-2404 (2009).

11. Jiang, Z. P., I. Mareels, and Y. Wang, "A Lyapunov formulation of the nonlinear small-gain theorem for interconnected ISS systems," Automatica, Vol. 32, pp. 1211-1215 (1996). 
12. Jiang, Z.P., A. R. Teel, and L. Praly. "Small-gain theorem for ISS systems and applications," Math. Control Signal Syst., Vol. 7, pp. 95-120 (1994).

13. Karafyllis, I. and Z. P. Jiang, "A new small-gain theorem with an application to the stabilization of the chemostat," Int. J. Robust Nonlinear Control, DOI: 10.1002/rnc.1773 (2011)

14. Sontag, E. D., "Smooth stabilization implies coprime factorization," IEEE Trans. Autom. Control, Vol. 34, pp. 435-443 (1989).

15. Sontag, E. D., "Comments on integral variants of ISS," Syst. Control Lett., Vol. 34, pp. 93-100 (1998).

16. Sontag, E. D. and Y. Wang, "On characterizations of input-to-state stability property," Syst. Control Lett., Vol. 24, pp. 351-359 (1995).

17. Teel, A, "A nonlinear small gain theorem for the analysis of control systems with saturation," IEEE Trans. Automat. Control, Vol. 41, pp. 1256-1270 (1996).

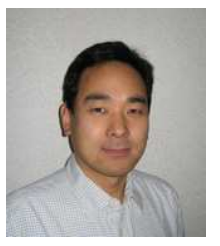

Hiroshi Ito received B.E., M.E., and Ph.D. degrees in electrical engineering from Keio University, Tokyo, Japan, in 1990, 1992, and 1995, respectively. From 1994 to 1995, he was Research Fellow of Japan Society for the Promotion of Science (JSPS). He has been with the Department of Control Engineering and Science, Kyushu Institute of Technology, Fukuoka, Japan, since 1995. He is currently Associate Professor at Department of Systems Design and Informatics. From 1998 to 1999, he held visiting researcher positions at Northwestern University and University of California, San Diego. His main research interests include stability of nonlinear systems, theory of robustness, time-delay systems, multi-rate sampled data control, asynchronous systems and large-scale systems with emphasis on applications to biological systems, communication networks, and smart grids dynamics. Dr. Ito received 1990 Young Author Prize of The
Society of Instrument and Control Engineers (SICE) and Pioneer Award of Control Division of SICE in 2008. He is also a recipient of SICE-ICCAS 2006 Best Paper Award and SICE 2008 International Award. He has served as Associate Editor of IEEE Transactions on Automatic Control. Currently, he is on IEEE CSS Conference Editorial Board.

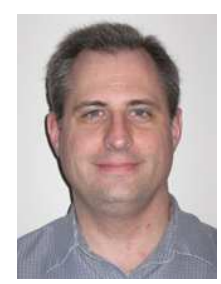

Randy A. Freeman received a Ph.D. in Electrical Engineering from University of California at Santa Barbara in 1995. Since then he has been a faculty member at Northwestern University (Evanston, Illinois), where he is currently Professor of Electrical Engineering and Computer Science. He received National Science Foundation CAREER Award in 1997. He has been a member of the IEEE Control System Society Conference Editorial Board since 1997, has served on Program and Operating Committees for American Control Conference and the IEEE Conference on Decision and Control, and has served Associate Editor of the IEEE Transactions on Automatic Control. His research interests include nonlinear system theory, nonlinear control, robust control, and optimal control.

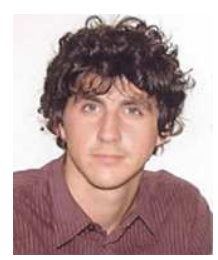

Antoine Chaillet is Associate Professor at University Paris Sud 11-Supélec-L2S since 2007. His scientific interests are the analysis and control of interconnected nonlinear dynamical systems. His research has found applications in neuroscience, vehicle formations, network controlled systems, and control of mechanical systems. He is the author of 13 publications in international journals, and more than 30 book chapters and international conference papers. He works as an expert for European Commission and French and Romanian research agencies. In 2010, he received the award "Prime d' Excellence Scientifique" from French ministry of research. 